\title{
The effect of nitric oxide inhalation on heart and pulmonary circulation in rabbits with acute massive pulmonary embolism
}

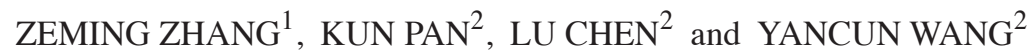 \\ ${ }^{1}$ Department of Respiratory Medicine, Affiliated Zhou Pu Hospital of Shanghai Health College, Shanghai 201318; \\ ${ }^{2}$ Department of Respiratory Medicine, Affiliated Hospital of Hebei University, Baoding, Hebei 071000, P.R. China
}

Received August 9, 2017; Accepted April 19, 2018

DOI: $10.3892 /$ etm.2018.6155

\begin{abstract}
The aim of the present study was to investigate the effect of nitric oxide inhalation (NOI) on cardiac troponin I (CTnI) levels and mean pulmonary arterial pressure (mPAP) in rabbits with acute massive pulmonary embolism (AMPE). Thirty rabbits were used as animal models for AMPE and received different treatments. A total of $4 \mathrm{~h}$ after successful modeling, the control group $(\mathrm{CON}, \mathrm{n}=10)$ received conventional thrombolysis, whereas the treatment group (TRE, $\mathrm{n}=10$ ) received conventional thrombolysis plus NOI. The experimental group (EXP, $n=10)$ did not receive any treatments. Myocardial necrosis was pathologically confirmed in all 30 rabbits. In group EXP, the post-AMPE CTnI peak level was $0.42 \pm 0.12 \mu \mathrm{g} / \mathrm{l}$, was achieved in $18.8 \pm 4.5 \mathrm{~h}$ and remained positive for $38.6 \pm 5.2 \mathrm{~h}(\geq 0.1 \mu \mathrm{g} / \mathrm{l})$. These values were lower in group TRE when compared with those in groups CON and EXP $(\mathrm{P}<0.05)$. Group TRE exhibited significantly reduced mPAP at $24,28,32$, and $34 \mathrm{~h}(\mathrm{P}<0.05)$ when compared with group CON. AMPE-induced cardiac impairment was more severe in group EXP when compared with groups CON and TRE. The present findings indicated that the CTnI peak was significantly correlated with the corresponding mPAP. Furthermore, the results suggested NOI may reduce mPAP and CTnI peak levels, with protective effects against AMPE-induced myocardial damage in rabbits.
\end{abstract}

\section{Introduction}

Early mortality in patients with acute massive pulmonary embolism (AMPE) is as high as $30-55 \%(1,2)$. Mortality can be even higher when accompanied by shock and heart failure $(3,4)$. The reasons for ventricular dysfunction and

Correspondence to: Dr Kun Pan, Department of Respiratory Medicine, Affiliated Hospital of Hebei University, 212 Yuhua East Road, Lianchi, Baoding, Hebei 071000, P.R. China

E-mail: cnkunpan@163.com

Key words: pulmonary thromboembolism, rabbits, treatment, nitric oxide inhalation, mean pulmonary arterial pressure failure in patients with PE remain unclear $(5,6)$. However, these patients are more likely to have myocardial damage and increased cardiac troponin I (CTnI) levels during heart failure $(7,8)$. Furthermore, the plasma troponin levels and extent of myocardial damage are significantly negatively correlated with the clinical prognosis in these patients (9). Therefore, investigating the causes and factors associated with myocardial damage in PE patients will aid in determining the clinical prognosis.

Since nitric oxide (NO) acts as a vasodilator (10-12), nitric oxide inhalation (NOI) is important for the treatment of massive pulmonary thrombosis (PT) $(13,14)$. NOI mitigates lung injury by reducing pulmonary hypertension and neutrophil migration, promotes endothelial integrity in the lung, improves pulmonary ventilation/perfusion, increases vascular density, and repairs vascular endothelial cells $(15,16)$.

$\mathrm{NO}$ is an important signaling molecule in the cardiovascular system and is considered to be a ubiquitous cardioprotective mediator (17). However, further research is required to determine whether NOI has a protective effect against myocardial damage in AMPE.

For further study of the mechanisms of AMPE, we prepared a rabbit model of AMPE and studied the release curve of CTnI, the relationship between CTnI and mean pulmonary arterial pressure (mPAP), and the impact of NOI on CTnI and mPAP.

\section{Materials and methods}

Animals and grouping. A total of 30 healthy Japanese white rabbits (not restricted by sex) aged 6 to 9 months (weight: 2-2.5 kg) were provided by the Laboratory Animal Center, Medical Department of Hebei University. They were randomly divided into an experimental group (EXP; $n=10)$ that did not receive any treatments, an AMPE control group $(\mathrm{CON} ; \mathrm{n}=10)$ that received urokinase (intravenous drip, $20,000 \mathrm{U} / \mathrm{kg} / 2 \mathrm{~h}$ ), and a treatment group (TRE; $\mathrm{n}=10$ ) that received conventional thrombolysis plus NOI. This study was carried out in strict accordance with the recommendations in the Guide for the Care and Use of Laboratory Animals of the National Institutes of Health. The animal use protocol has been reviewed and approved by the Institutional Animal Care and Use Committee (IACUC) of Hebei University. 
Preparation of the MPE animal model

Blood sampling and preparation of artificial thrombus. Venous blood $(2 \mathrm{ml})$ was sampled from the ear vein of each rabbit using an indwelling needle and was allowed to settle in a sterile culture dish at room temperature for $15 \mathrm{~min}$. This was then placed in a constant-temperature water bath $\left(70^{\circ} \mathrm{C}\right)$ for $30 \mathrm{~min}$ to promote blood clotting. Thrombi were then disinfected and cut into $1 \times 7 \times 1 \mathrm{~mm}$ emboli.

Under sterile conditions, anesthetic (20\% urethane solution, $1.0 \mathrm{~g} / \mathrm{kg}$ ) was slowly injected into the ear vein of each rabbit. Rabbits were then prepared for a $2-\mathrm{cm}$ conventional transverse incision in the anterior chest for tracheal intubation to protect the airway for spontaneous breathing and mechanical ventilation. After the right jugular vein and left external carotid artery were separated, while simultaneously exposing the left femoral vein, a homemade pulmonary artery catheter and a microvascular catheter (5-Fr TIG; Tyler Company, Japan) were inserted into the right jugular vein and left carotid artery, respectively. An oscilloscope was used for monitoring during placement in order to determine the positions of the catheter tips as indicated by high pressure waveforms, which reflected the length of pulmonary catheter insertion. During the experiment, physiological saline $(0.3 \mathrm{ml} / \mathrm{min})$ was continuously instilled through the pulmonary artery catheters using a microperistaltic pump. The two catheters were connected to a multi-channel physiological parameter analyzer (MP150; Biopac Systems, Inc., Goleta, CA, USA) through a pressure sensor. The mean arterial pressure and mPAP were controlled synchronously. The left femoral vein was also punctured for placement of a microcatheter for in-time rehydration and blood sampling. The prepared emboli ( $0.5 \mathrm{ml}$ per injection) were then repeatedly injected (time interval=3 $\mathrm{min}$ ) into the right jugular vein via the pulmonary artery catheter using a 5-ml syringe, followed by an infusion of physiological saline $(2 \mathrm{ml})$, until the mPAP increased to $45 \mathrm{mmHg}$, the mean arterial blood pressure decreased to approximately $20-40 \%$ of baseline, and hypotension was achieved and maintained (approximately 55-60 mmHg blood pressure). AMPE modeling was determined to be successful when symptoms such as shortness of breath or dyspnea occurred and lasted for approximately $40 \mathrm{~min}$, at which time the injection of emboli was terminated. During modeling, if severe dyspnea developed such that the process could not continue, short-term mechanical ventilation (SERVO-i infant ventilator, Rontgenvagen2, SE-17154Solna; Siemens Maquet Critical Care AB, Slona, Sweden) was performed (pressure: $20 \mathrm{cmH}_{2} \mathrm{O}$, respiratory rate: 28 breaths/min).

Perfusion and observation of the results. When the experiment was completed after $48 \mathrm{~h}$ of successful modeling, the rabbits were perfused with ABS solution, with BIOPACK pressure monitoring (18-25 $\mathrm{mmHg})$. The specific procedures were as follows:

Anesthesia. The rabbit ear vein runs along the trailing edge. The fur covering the skin over the vein was pulled or cut off, and the skin was moistened with water. The vein was slightly rubbed or flicked using fingers to increase blood flow, then compressed at the ear root until the vein became engorged.
After injection, the needle was pulled out and a cotton ball was used to apply pressure at the incision to stop bleeding.

Fixation. Each rabbit was placed in the prone position on a laboratory table, with the limbs and jaws tightly fixed.

Preoperative preparation. Fur was removed from the chest and abdomen, which were then coated with a layer of hair removal cream (using a medical cotton swab) for approximately $10 \mathrm{sec}$, and gently wiped using wet gauze until the fur was completely removed. Meanwhile, an ABS solution $(80 \mathrm{ml})$ was prepared for future use.

Surgery A. A 6-cm incision was made along the linea alba to expose the abdominal organs. The abdominal aorta was then gradually exposed, freed, and threaded for further use. The prepared heparin saline was then connected to the infusion device and needle. The needle was cut and the abdominal aorta was cannulated, followed by knotting and fixation. The inferior vena cava was cut short, and the infusion device was opened to rinse the blood vessels.

Surgery $B$. When the liver appeared pale after washing, heparin-rinsing was stopped and $50 \mathrm{ml}$ of acetone was slowly instilled until the lung tissue exhibited a color change and the inferior vena cava emitted a strong odor. The thoracic cavity and manubrium were then opened in order to ligate the superior vena cava. An inferior vena cava catheter was inserted above the diaphragm and connected to a BIOPACK multi-function pressure detector and the prepared ABS perfusion fluid. This was used to inject the perfusion liquid until the pressure rose to a point where it could not be reduced $(18-25 \mathrm{mmHg})$, at which time the injection was stopped.

The specimen was then removed for micro-computed tomography (MicroCT; A00001514J; PerkinElmer, Inc., Yokohama, Japan). The lung and heart tissues were taken. After fixation with formaldehyde (Sigma-Aldrich; Merck KGaA, Darmstadt, Germany) and embedding with paraffin (Sigma-Aldrich; Merck KGaA), the tissue slices with thickness of $5 \mu \mathrm{m}$ were prepared. The routine H\&E staining was performed (Sigma-Aldrich; Merck KGaA). The Histomorphological changes were observed under DVM6 optical microscope (Leica Science Lab, Leica Camera AG Berlin, Germany). The sections with flaky aggregation of necrosis were defined as positive (Fig. 1), and the rest were negative.

Groups CON and TRE underwent thrombolysis or thrombolysis+NOI $4 \mathrm{~h}$ after modeling. Simultaneously, mechanical ventilation was also applied with the concentration of inhaled oxygen at $50 \%$.

\section{NOI}

Method of NOI. After successful modeling, defined as when the mPAP of the rabbit reached $40 \%$, and the rabbit developed shortness of breath or dyspnea for $4 \mathrm{~h}$, mechanical ventilation was initiated. NO comprised 800 ppm of decompressed NO, $\mathrm{N}_{2}$, and air. When the concentration of NOI reached 10-20 ppm, a ventilator pipe was connected to the NOI pipe for invasive mechanical ventilation; the ventilator was set to pressure control mode (pressure: $20 \mathrm{cmH}_{2} \mathrm{O}$, respiratory rate: 28 breaths/min, 


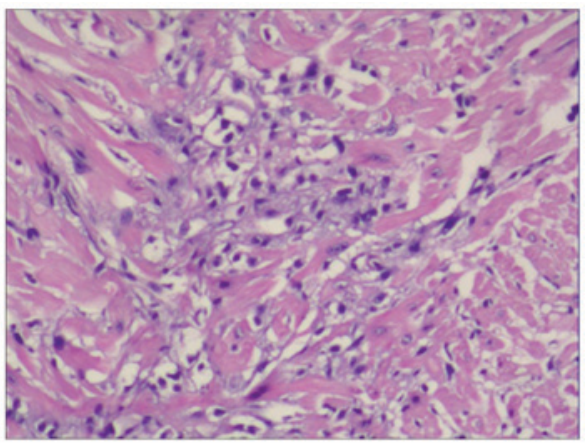

Figure 1. H\&E staining of myocardium. The arrow indicated the flaky aggregation of myocardial necrosis (magnification, x200).

oxygen concentration for mechanical ventilation: $50 \%$ ). NOI was administered via a delivery system (Datex-Ohmeda, Madison, WI, USA). The level of methemoglobin was also monitored during the experiment and kept at $<0.3 \mathrm{~g} / \mathrm{l}$.

Blood collection and determination of CTnI. Blood was sampled before treatment and every $4 \mathrm{~h}$ after treatment. The mean arterial pressure and mPAP were also monitored simultaneously.

The concentration of CTnI was determined using a microparticle chemiluminescence method and an automatic immunoassay analyzer. The kit was provided by Beckman Coulter, Inc. (Brea, CA, USA).

Statistical analysis. The data were expressed as means \pm standard deviation and were processed using SPSS for Windows, Version 21.0 (IBM SPSS., Armonk, NY, USA). Intergroup comparisons of Indices were performed using single-factor analysis of variance with Tukey's post hoc test and paired comparisons were performed with the q test. The pathological parameters were expressed by the positive rate, and were analyzed using Fisher's test. $\mathrm{P}<0.05$ was considered to indicate a statistically significant difference.

\section{Results}

All 30 rabbits were successfully adapted to the MPE model using this method (confirmed with MicroCT), with a $100 \%$ success rate (Fig. 2).

Pulmonary histopathological changes in the AMPE model. Histopathology revealed that the embolized pulmonary region was pale and ischemic; the non-embolized region is shown in Fig. 3A. The pale region is shown in Fig. 3B, in which large vascular emboli can be seen, and small vascular emboli are shown in Fig. 3C (microscopy); the bleeding conditions around the embolized lung tissue are shown in Fig. 3D.

Cardiac histopathology. The right ventricle was enlarged, vasodilated, and congested (Fig. 4A), and the right ventricular wall became thinner (Fig. 4B and C); microscopically, the left ventricle exhibited myocardial necrosis (Fig. 4D).

In 30 pathological slices in the EXP group, there were 27 slices of obvious myocardial necrosis. After thrombolysis,

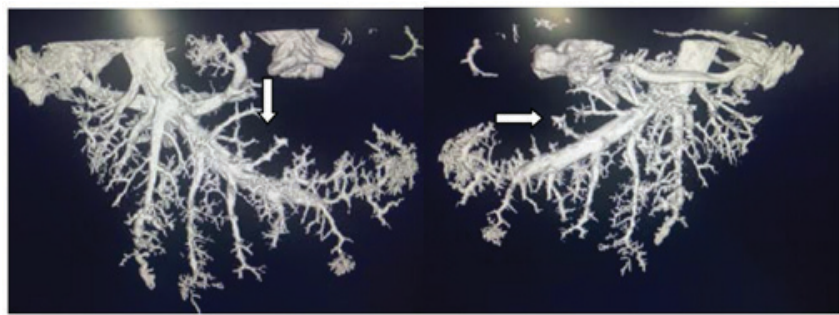

Figure 2. MPE model MicroCT imaging. Arrows indicated the embolized pulmonary vessel. MPE, massive pulmonary embolism; MicroCT, micro-computed tomography.

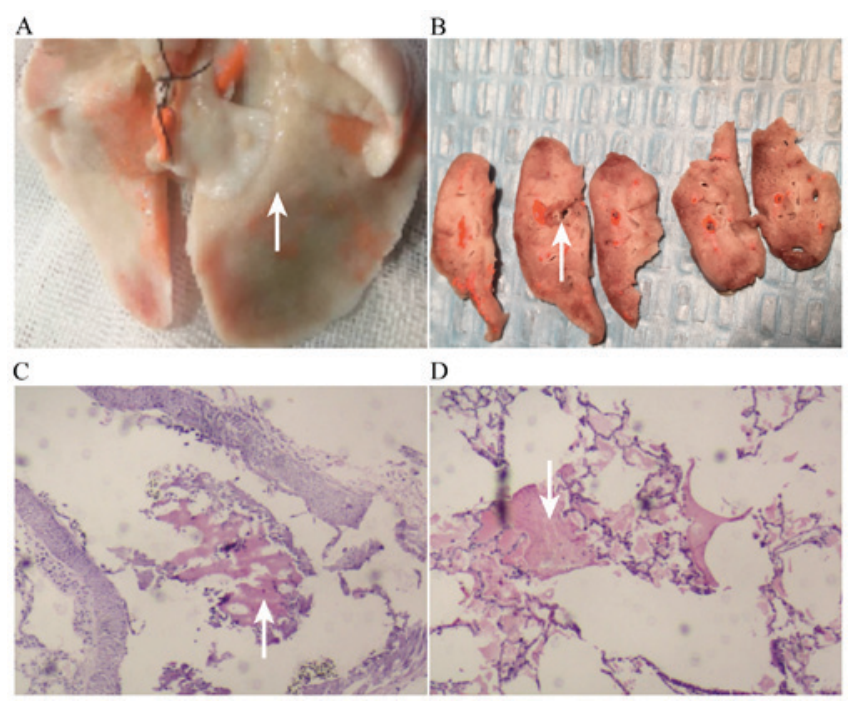

Figure 3. Pulmonary histopathological changes in the AMPE model. (A) Pulmonary gross anatomy. (B) Anatomy along the pale site revealed embolized pulmonary artery; (C) Emboli in the arteriola under light microscope (H\&E, magnification, $x 100)$; (D). Bleeding in the pulmonary tissue under light microscope (H\&E magnification, x100). Arrows indicated the embolized pale ischemic site. AMPE, acute massive pulmonary embolism.

myocardial necrosis was still obviously increased, with no significant difference with the EXP group. The emboli could be seen intravascularly (Fig. 4D). Thrombolysis+NOI significantly reduced myocardial necrosis. In 30 pathological slices, there were 8 slices of obvious myocardial necrosis, with positive rate of $27 \%$. Only scattered necrotic areas could be seen, together with intravascular emboli (Fig. 4E). High-power microscopy revealed more obvious changes in group TRE; myocardial necrosis could be clearly seen after thrombolysis (Fig. 4F). Thrombolysis+NOI scattered necrosis was seen (Fig. 4G and Table I).

Concentration curve of CtnI. The curve of CTnI became positive $4 \mathrm{~h}$ after modeling, peaked at $18.8 \pm 4.5 \mathrm{~h}$, and remained positive for $38 \pm 5.2 \mathrm{~h}$; the peak CTnI concentration was $0.42 \pm 0.12 \mu \mathrm{g} / 1$ (Fig. 5).

The peak CTnI concentration and mPAP were significantly correlated in rabbits with AMPE (r=0.98, $\mathrm{P}<0.05$; Fig. 6).

CTnI peak time and sustained positive duration. The CTnI peak time and sustained positive duration were significantly longer and the plasma peak concentration in group EXP was significantly higher when compared with groups CON and 
Table I. Slices with obvious myocardial necrosis in different groups.

\begin{tabular}{|c|c|c|c|}
\hline Group (n) & Positive, n (\%) & Negative, n (\%) & P-values \\
\hline EXP (30) & $27(90)$ & $3(10)$ & 0.007 (EXP vs. CON) \\
\hline CON (30) & $18(60)$ & $12(40)$ & 0.038 (CON vs. TRE) \\
\hline TRE (30) & $10(33)$ & $20(67)$ & $<0.001$ (EXP vs. TRE) \\
\hline
\end{tabular}

Positive indicated the slice was identified with obvious myocardial necrosis, whereas negative indicated no myocardial necrosis. EXP, experimental group; CON, control group; TRE, treatment group.

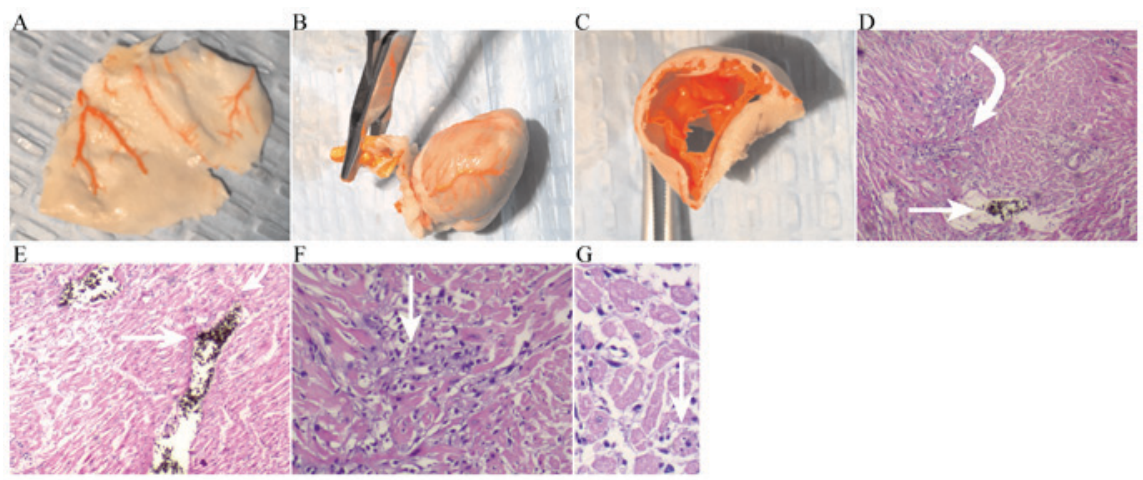

Figure 4. Cardiac histopathological changes in the AMPE model. (A) The right ventricle was enlarged; (B). the right ventricular wall became thinner; (C). the ventricular wall on the cross section became thinner; (D) there were large area of necrotic myocardium in the model group (H\&E magnification, x100), (E) Thrombolysis+NOI significantly reduced myocardial necrosis, and only scattered myocardial necrosis was observed (H\&E magnification, $\mathrm{x} 100$ ). (F) A large area of myocardial necrosis was revealed following thrombolysis (arrow indicated clumps of myocardial necrosis) (H\&E magnification, x200). (G) Thrombolysis+NOI markedly reduced myocardial necrosis. Arrow indicated scattered myocardial necrosis (H\&E, magnification, x100). AMPE, acute massive pulmonary embolism; NOI, nitric oxide inhalation.

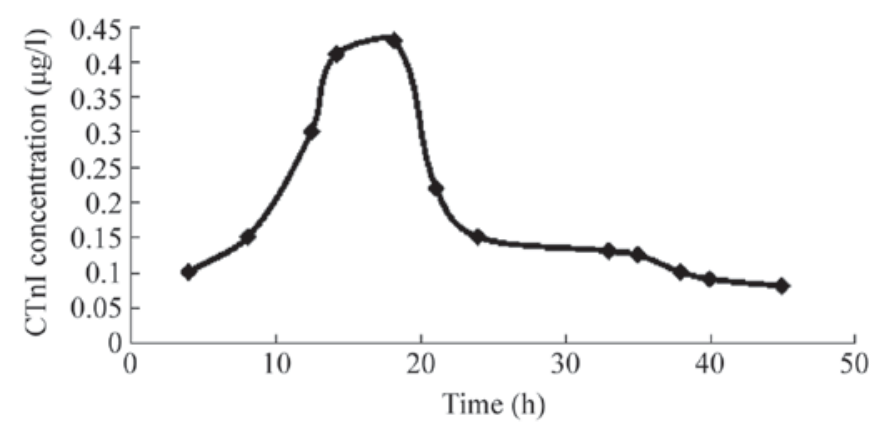

Figure 5. CTnI over time curve. CTnI concentration increased from $4 \mathrm{~h}$ after modeling, peaked at $18 \mathrm{~h}$ and then decreased until $>40 \mathrm{~h}$. CTnI, cardiac troponin I.

TRE $(\mathrm{P}<0.047, \mathrm{P}<0.03)$; corresponding durations in group CON were significantly longer when compared to those in group TRE $(\mathrm{P}<0.048, \mathrm{P}<0.036)$.

The CTnI peak concentration in group EXP was significantly higher than that in group CON $(\mathrm{P}<0.039)$, and that of group CON was also significantly higher than that of group TRE $(\mathrm{P}<0.014$; Table II).

$m P A P$. The post-modeling mPAP in group EXP slowly increased with prolongation of modeling time. The post-modeling mPAP in group CON gradually increased after thrombolysis, but gradually decreased after peaking. The post-modeling mPAP in group TRE increased slowly after

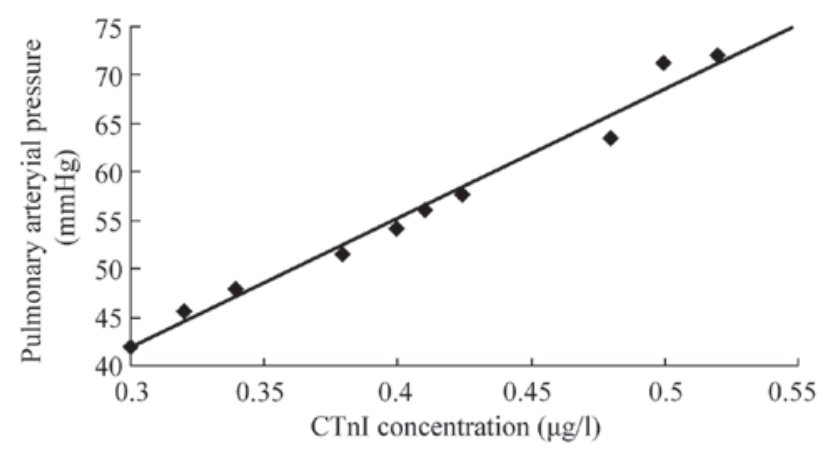

Figure 6. Correlation analysis of CTnI and pulmonary arterial pressure. There is a clear linear positive correlation between the pulmonary arterial pressure and CTnI. CTnI, cardiac troponin I.

treatment, then decreased, and was significantly different at $24 \mathrm{~h}(63.5 \pm 5.9,58.1 \pm 5.5, \mathrm{P}<0.04), 28 \mathrm{~h}(61.2 \pm 5.7,55.3 \pm 5.6$, $\mathrm{P}<0.03), 32 \mathrm{~h}(57.6 \pm 5.4,52.5 \pm 5.3, \mathrm{P}<0.05)$, and $34 \mathrm{~h}(55.4 \pm 4.3$, $51.3 \pm 4.2, \mathrm{P}<0.04)$ in group TRE when compared with group CON (Fig. 7).

\section{Discussion}

$\mathrm{PE}$ is common and is characterized by various clinical manifestations. Patients with AMPE have increased mortality and poor prognosis, which may be associated with myocardial damage. Studies have confirmed that CTnI is increased in PT, with peak concentration showing a significant correlation 
Table II. Comparison of CTnI changes among the three groups.

\begin{tabular}{|c|c|c|c|c|}
\hline \multirow[b]{2}{*}{ Group (n) } & \multicolumn{4}{|c|}{$\mathrm{CTnI} \geq 0.1 \mu \mathrm{g} / \mathrm{l}^{\mathrm{a}}$} \\
\hline & $\begin{array}{l}\text { Positive rate } \\
{[4 \mathrm{~h}, \mathrm{n}(\%)]}\end{array}$ & $\begin{array}{l}\mathrm{CTnI} \\
\text { peaking time }(\mathrm{h})\end{array}$ & $\begin{array}{c}\text { Positive } \\
\text { duration (h) }\end{array}$ & $\begin{array}{c}\text { Plasma peak } \\
\text { concentration }(\mu \mathrm{g} / \mathrm{l})\end{array}$ \\
\hline EXP 10 & $10 / 10(100)$ & $18.81 \pm 4.51$ & $38.62 \pm 5.22$ & $0.42 \pm 0.12$ \\
\hline CON 10 & 10/10 (100) & $15.13 \pm 3.21^{\mathrm{b}}$ & $34.10 \pm 3.53^{\mathrm{b}}$ & $0.31 \pm 0.10^{\mathrm{b}}$ \\
\hline TRE 10 & 10/10 (100) & $12.42 \pm 2.43^{\mathrm{c}}$ & $31.04 \pm 2.22^{\mathrm{c}}$ & $0.21 \pm 0.06^{c}$ \\
\hline EXP vs. CON & & $<0.05$ & $<0.03$ & $<0.01$ \\
\hline TRE vs. CON & & $<0.05$ & $<0.04$ & $<0.04$ \\
\hline
\end{tabular}

${ }^{\mathrm{a} C T n I} \geq 0.1 \mu \mathrm{g} / \mathrm{l}$ is defined as CTnI-positive; ${ }^{\mathrm{b}}$ compared with group EXP; ${ }^{\mathrm{c}}$ compared with group CON. EXP, experimental group; CON, control group; TRE, treatment group; CTnI, cardiac troponin I.

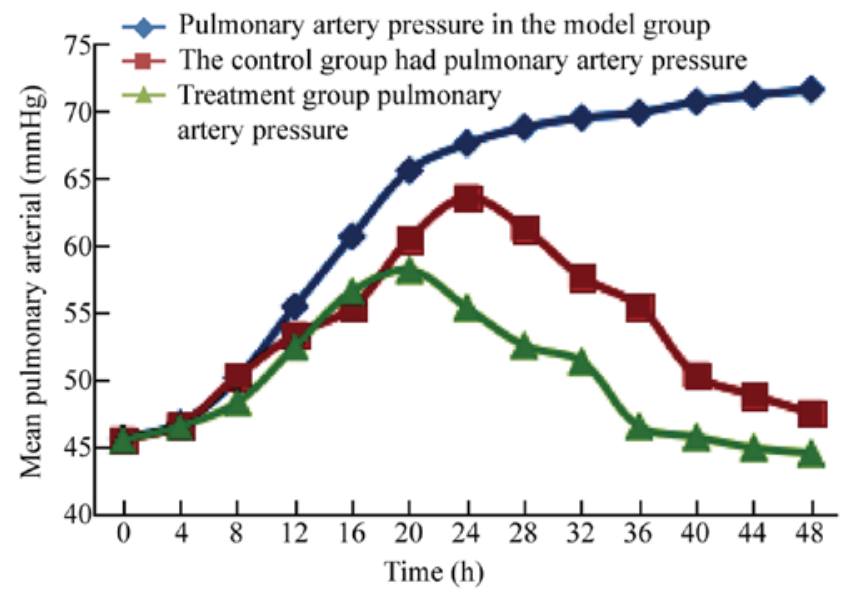

Figure 7. Mean pulmonary arterial curve. In group EXP, mPAP increased; in group CON, mPAP increased after thrombolysis then decreased after peaking; in group TRE, mPAP increased slowly after treatment, then decreased. EXP, experimental group; CON, control group; TRE, treatment group; mPAP, mean pulmonary arterial pressure.

with mortality (15). Therefore, further investigate the status of impaired myocardium in PT, it is necessary to establish a PT-induced myocardial injury model. Our previous studies confirmed that directly injecting emboli into the pulmonary artery can rapidly increase pulmonary artery pressure, thus rapidly leading to myocardial necrosis; therefore, this method has a higher success rate for creating an AMPE model. Studies have shown that all rabbits with AMPE have accompanying myocardial damage, which is also associated with the formation of intravascular emboli. This has been confirmed by our pathological results. Therefore, the levels of CTnI in the rabbit model are significantly higher than normal $(0.1 \mu \mathrm{g} / 1)$. Our studies also confirmed that CTnI becomes elevated $4 \mathrm{~h}$ after establishing the MPE model, peaks at $18.8 \pm 4.5 \mathrm{~h}$, and remains elevated for $38.6 \pm 5.2 \mathrm{~h}$. This differs from the release curve in patients with myocardial infarction, who exhibit an elevated CTnI $3 \mathrm{~h}$ after onset of symptoms, with the level remaining elevated for 10 to 14 days. Characteristic features include the late appearance of CTnI peak concentration and short duration of elevation, with no fluctuations. These features indicate that the mechanism of PT-induced myocardial damage is different from that in myocardial infarction-induced release of CTnI into the blood $(16,17)$.

In our study, the CTnI released into the blood in rabbits with MPE showed no significant correlation with the mean arterial pressure in the systemic circulation, indicating that myocardial ischemia and hypoxia-induced myocardial damage, which are caused by the reduction of average arterial pressure, are not the main cause of PT. This is consistent with previous studies showing that when MPE occurs, blood pressure decreases, but does not reduce coronary blood flow; therefore, there is no myocardial damage (18).

In our study, the CTnI peak concentration in AMPE was significantly correlated with the mPAP at the same time points, indicating that pulmonary hypertension plays a major role in the process of myocardial damage in rabbits with $\operatorname{MPE}(19,20)$. It has been reported that when pulmonary arterial embolization occurs, platelet dysfunction also occurs, which then activates platelets and significantly increases thromboxane (TXA2) levels, thus leading to heart failure and circulatory shock. These vasoactive substances may partially account for myocardial damage in PT. However, the CTnI peak concentration is significantly correlated with the mPAP at the same time points, indicating that the increase in mPAP plays a major role in determining the degree of myocardial damage in AMPE $(21,22)$. Therefore, in PT, mechanical obstruction and vasoactive factors can lead to pulmonary hypertension. This increases the right ventricular post-load, which results in an increase in right ventricular work, causing myocardial ischemia and hypoxia; therefore, this plays an important role in the extent of myocardial damage and, in turn, patient prognosis. This is consistent with our findings. Our study showed that the pulmonary arteries in rabbits with MPE were widened and the right ventricle was enlarged, but the right ventricular wall became thinner. Although the mechanism of PT-induced myocardial damage is not very clear (18), it has been reported that the left and right ventricles are both involved (21). This is consistent with our findings. Combined with our studies, it can be deduced that the left and right ventricles are both involved; the right ventricle may mainly exhibit mechanical traction damage caused by the pulmonary hypertension-induced right ventricular post-load increase and thinning of the 
ventricular wall. However, the damage in the left ventricle may be mainly related to PT-induced platelet dysfunction, which activates platelets and significantly increases TXA2 levels. This causes cardiac vascular damage, intravascular thrombosis, myocardial ischemia/hypoxia, and myocardial necrosis. The above pathological changes have also been confirmed by our results.

After successfully creating the animal model, the mPAP increased slowly and remained at a high level within $48 \mathrm{~h}$ of modeling. This phenomenon is another indication that the development of pulmonary hypertension is not simply due to mechanical obstruction. As disease progresses, vasoactive factors play certain roles in the development of pulmonary hypertension. Hence, simple thrombolysis cannot immediately decrease the mPAP. With time, the mPAP increases to a peak, followed by a significant decrease. This may be caused by the shorter thrombolysis time; furthermore, when the emboli dissolve, the roles of vasoactive factors also gradually decrease (23).

Thrombolysis+NOI exhibited a similar change in the mPAP curve as a result of simple thrombolysis. However, with time, the mPAP increased slowly. This was mainly due to the role of NOI (24). NO is an endogenous factor that promotes vascular smooth muscle relaxation (25) with an obvious and rapid effect. Thus, the changes in the mPAP curves in groups TRE and CON are very similar. However, the mPAP changes at $24,28,32$, and $34 \mathrm{~h}$ showed statistical significance between the two groups, indicating that NOI can significantly reduce pulmonary hypertension in AMPE, and that thrombolysis and NOI synergistically reduce pulmonary hypertension in AMPE.

A comparison of cardiac damage between groups TRE and CON shows that these two treatment methods can both shorten the CTnI peak time and duration of elevation, as well as reduce the CTnI peak concentration. This indicates that thrombolysis and NOI individually and synergistically have protective effects against MPE-induced myocardial damage; the mechanism of the latter is related to the fact that NO can significantly reduce pulmonary hypertension, restore platelet function, and reduce TXA2 levels (26).

In conclusion, pulmonary hypertension is not an independent factor, but is still a major factor leading to myocardial damage in AMPE. NOI can significantly reduce pulmonary hypertension and protect against myocardial damage, and exhibits a synergistic effect with thrombolysis.

\section{Acknowledgements}

The authors would like to thank Professor Baoyuan Chen of the Department of Respiratory, Tianjin Medical University General Hospital (Tianjin China) for helping in writing this manuscript.

\section{Funding}

No funding was received.

\section{Availability of data and materials}

The datasets used and/or analyzed during the current study are available from the corresponding author on reasonable request.

\section{Authors' contributions}

$\mathrm{ZZ}$ and KP participated in the design of this study, and ZZ, LC and YW performed the experiments and collected the data. LC performed the statistical analysis. ZZ and YW collected important background information. ZZ, KP and LC drafted the manuscript. All authors read and approved the final manuscript.

\section{Ethics approval and consent to participate}

This study was carried out in strict accordance with the recommendations in the Guide for the Care and Use of Laboratory Animals of the National Institutes of Health. The animal use protocol has been reviewed and approved by the Institutional Animal Care and Use Committee (IACUC) of Hebei University.

\section{Consent for publication}

Not applicable.

\section{Competing interests}

The authors declare that they have no competing interests.

\section{References}

1. Darze ES, Casqueiro JB, Ciuffo LA, Santos JM, Magalhães IR and Latado AL: Pulmonary embolism mortality in Brazil from 1989 to 2010: Gender and regional disparities. Arq Bras Cardiol 106: 4-12, 2016

2. Yavuz S, Toktas F, Goncu T, Eris C, Gucu A, Ay D, Erdolu B, Tenekecioglu E, Karaagac K, Vural H and Ozyazicioglu A: Surgical embolectomy for acute massive pulmonary embolism. Int J Clin Exp Med 7: 5362-5375, 2014.

3. Dalen JE and Alpert JS: Natural history of pulmonary embolism. Prog Cardiovasc Dis 17: 259-270, 1975.

4. Soloff A and Rodman T: Acute pulmonary embolism. II. Clinical. Am Heart J 74: 829-847, 1967.

5. Jones AE, Watts JA, Debelak JP, Thornton LR, Younger JG and Kline JA: Inhibition of prostaglandin synthesis during polystyrene microsphere-induced pulmonary embolism in the rat. Am J Physiol Lung Cell Mol Physiol 284: L1072-L1081, 2003.

6. Cho JH, Kutti Sridharan G, Kim SH, Kaw R, Abburi T, Irfan A and Kocheril AG: Right ventricular dysfunction as an echocardiographic prognostic factor in hemodynamically stable patients with acute pulmonary embolism: A meta-analysis. BMC Cardiovasc Disord 14: 64, 2014.

7. Meyer T, Binder L, Hruska N, Luthe $\mathrm{H}$ and Buchwald AB: Cardiac troponin I elevation in acute pulmonary embolism is associated with right ventricular dysfunction. J Am Coll Cardiol 36: 1632-1636, 2000.

8. French Intensive Care Society, International congress-Réanimation 2016. Ann Intensive Care 6 (Suppl 1): S50, 2016.

9. Kreit JW: The impact of right ventricular dysfunction on the prognosis and therapy of normotensive patients with pulmonary embolism. Chest 125: 1539-1545, 2004.

10. Kline JA, Hernandez J, Garrett JS and Jones AE: Pilot study of a protocol to administer inhaled nitric oxide to treat severe acute submassive pulmonary embolism. Emerg Med J 31: 459-462, 2014.

11. Trummer G, Berchtold-Herz M, Martin J and Beyersdorf F: Successful treatment of pulmonary hypertension with inhaled nitric oxide after pulmonary embolectomy. Ann Thorac Surg 73: 1299-1301, 2012

12. Waldow T, Witt W, Janke A, Ulmer A, Buzin A and Matschke K: Cell-cell junctions and vascular endothelial growth factor in rat lung as affected by ischemia/reperfusion and preconditioning with inhaled nitric oxide. J Surg Res 157: 30-42, 2009. 
13. Qi Y, Qian L, Sun B, Liu L, Wu P and Sun L: Inhaled NO contributes to lung repair in piglets with acute respiratory distress syndrome via increasing circulating endothelial progenitor cells. PLoS One 7: e33859, 2012.

14. Strijdom H, Chamane $N$ and Lochner A: Nitric oxide in the cardiovascular system: A simple molecule with complex actions. Cardiovasc J Afr 20: 303-310, 2009.

15. Elias A, Mallett S, Daoud-Elias M, Poggi JN and Clarke M: Prognostic models in acute pulmonary embolism: A systematic review and meta-analysis. BMJ Open 6: e010324, 2016.

16. Müller-Bardorff M, Weidtmann B, Giannitsis E, Kurowski V and Katus HA: Release kinetics of cardiac troponin $\mathrm{T}$ in survivors of confirmed severe pulmonary embolism. Clin Chem 48: 673-675, 2002.

17. Go AS, Mozaffarian D, Roger VL, Benjamin EJ, Berry JD, Blaha MJ, Dai S, Ford ES, Fox CS, Franco S, et al: Heart disease and stroke statistics-2014 update: A report from the American Heart Association. Circulation 129: e28-e292, 2014.

18. Zagorski J, Gellar MA, Obraztsova M, Kline JA and Watts JA: Inhibition of CINC-1 decreases right ventricular damage caused by experimental pulmonary embolism in rats. J Immunol 179: 7820-7826, 2007.

19. Lannan KL, Phipps RP and White RJ: Thrombosis, platelets, microparticles and PAH: More than clot. Drug Discov Today 19: 1230-1235, 2014

20. von Brühl ML, Stark K, Steinhart A, Chandraratne S, Konrad I, Lorenz M, Khandoga A, Tirniceriu A, Coletti R, Köllnberger M, et al: Monocytes, neutrophils, and platelets cooperate to initiate and propagate venous thrombosis in mice in vivo. J Exp Med 209: 819-835, 2012.
21. Sullivan DM, Watts JA and Kline JA: Biventricular cardiac dysfunction after acute massive pulmonary embolism in the rat. J Appl Physiol (1985) 90: 1648-1656, 2001.

22. Montani D, Günther S, Dorfmüller P,Perros F, Girerd B, Garcia G, Jaïs X, Savale L, Artaud-Macari E, Price LC, et al: Pulmonary arterial hypertension. Orphanet J Rare Dis 8: 97, 2013.

23. Xu Q, Huang K, Zhai Z, Yang Y, Wang J and Wang C: Initial thrombolysis treatment compared with anticoagulation for acute intermediate-risk pulmonary embolism: A meta-analysis. J Thorac Dis 7: 810-821, 2015.

24. Tanus-Santos JE and Moreno H Jr: The use of inhaled nitric oxide during gas embolism. Chest 115: 1220-1221, 1999.

25. Yang Y, Feng Y, Zhou XG, Pan JJ and Zhou XY: Inhaled nitric oxide in preterm infants: An updated meta-analysis. J Res Med Sci 21: 41, 2016.

26. Nong Z, Hoylaerts M, Van Pelt N, Collen D and Janssens S Nitric oxide inhalation inhibits platelet aggregation and platelet-mediated pulmonary thrombosis in rats. Circ Res 81: 865-869, 1997. International (CC BY-NC-ND 4.0) License. 